\title{
Anti-human GITR Monoclonal Antibody TRX518
}

National Cancer Institute

\section{Source}

National Cancer Institute. Anti-human GITR Monoclonal Antibody TRX518. NCI

Thesaurus. Code 195023.

\begin{abstract}
A humanized, Fc disabled anti-human g lucocorticoid-induced tumor necrosis factor receptor (GITR) monoclonal antibody (MoAb) with immunomodulating activity. Antihuman GITR MoAb TRX518 blocks the interaction of GITR, found on multiple types of T cells, with its lig and, thereby inducing both the activation of tumor-antigen-specific $T$ effector cells, as well as abrogating the suppression induced by inappropriately activated T regulatory cells. This agent is shown to act synergistically with chemotherapeutic drugs in multiple cancer models.
\end{abstract}

